\title{
Effects of Increased Image Noise on Image Quality and Quantitative Interpretation in Brain CT Perfusion
}

K. Juluru, J.C. Shih, A. Raj, J.P. Comunale, H. Delaney, E.D. Greenberg, C. Hermann, Y.B. Liu, A. Hoelscher, N. Al-Khori, and P.C. Sanelli

\begin{abstract}
BACKGROUND AND PURPOSE: There is a desire within many institutions to reduce the radiation dose in CTP examinations. The purpose of this study was to simulate dose reduction through the addition of noise in brain $\mathrm{CT}$ perfusion examinations and to determine the subsequent effects on quality and quantitative interpretation.
\end{abstract}

MATERIALS AND METHODS: A total of 22 consecutive reference CTP scans were identified from an institutional review board-approved prospective clinical trial, all performed at $80 \mathrm{keV}$ and $190 \mathrm{mAs}$. Lower-dose scans at 188, 177, 167, 127, and $44 \mathrm{mAs}$ were generated through the addition of spatially correlated noise to the reference scans. A standard software package was used to generate CBF, CBV, and MTT maps. Six blinded radiologists determined quality scores of simulated scans on a Likert scale. Quantitative differences were calculated.

RESULTS: For qualitative analysis, the correlation coefficients for CBF $(-0.34 ; P<.0001)$, CBV $(-0.35 ; P<.0001)$, and MTT $(-0.44 ; P<$ .0001 ) were statistically significant. Interobserver agreements in quality for the simulated 188-, 177-, 167-, 127-, and 44-mAs scans for CBF were $0.95,0.98,0.98,0.95$, and 0.52 , respectively. Interobserver agreements in quality for the simulated CBV were 1, 1, 1, 1, and 0.83, respectively. For MTT, the interobserver agreements were $0.83,0.86,0.88,0.74$, and 0.05 , respectively. For quantitative analysis, only the lowest simulated dose of $44 \mathrm{mAs}$ showed statistically significant differences from the reference scan values for $\mathrm{CBF}(-1.8 ; P=.04)$, CBV $(0.07 ; P<.0001)$, and MTT $(0.46 ; P<.0001)$.

CONCLUSIONS: From a reference CTP study performed at $80 \mathrm{keV}$ and $190 \mathrm{mAs}$, this simulation study demonstrates the potential of a $33 \%$ reduction in tube current and dose while maintaining image quality and quantitative interpretations. This work can be used to inform future studies by using true, nonsimulated scans.

ABBREVIATIONS: $\quad$ ALARA $=$ as low as reasonably achievable; $C T D I-v o l=C T$ dose index volume; PABAK $=$ prevalence-adjusted bias-adjusted $\kappa$ values

$\mathbf{C}^{\mathrm{T}}$

$\mathrm{TP}$ is an imaging technique that allows rapid, noninvasive, quantitative evaluation of the hemodynamic status of the brain by generating parametric maps of CBF, CBV, and MTT. Frequently described applications of CTP in the literature include assessment of acute stroke, ${ }^{1-4}$ delayed cerebral ischemia related to vasospasm, ${ }^{5-8}$ and brain tumor imaging. ${ }^{9-12}$ The technique involves intravenous contrast injection and cine scanning with repetitive imaging through several sections of the brain to track the first pass of the contrast bolus. High radiation doses associated

Received June 29, 2012; accepted after revision October 22.

From the Department of Radiology, Weill Cornell Medical College, New YorkPresbyterian Hospital, New York, New York.

Please address correspondence to Krishna Juluru, MD, Weill Cornell Medical College, 525 E. 68th St, F-056, New York, NY 10065; e-mail: kjuluru@med.cornell.edu

- Indicates open access to non-subscribers at www.ajnr.org

$\checkmark$ Indicates article with supplemental on-line figures

http://dx.doi.org/10.3174/ajnr.A3448 with this technique, often performed in combination with noncontrast CT and CT angiography, have prompted notifications by the US Food and Drug Administration that stress the importance of proper acquisition parameters.

As a response to these concerns, the radiology community has heightened global efforts to reduce radiation dose in a variety of radiologic examinations, and CTP in particular, by optimizing imaging protocols under the ALARA principle. Major factors affecting radiation dose during a CT study include tube current, tube rotation time, peak voltage, pitch, and collimation. ${ }^{13}$ Changes in all of these parameters have various effects on image quality. Tube current is directly proportional to dose, and reductions in tube current primarily affect image noise without notable effects on other metrics of image quality such as image contrast.

Most CTP techniques have a fixed peak voltage at $80 \mathrm{kVp}$ to optimize image contrast but vary in tube current, with some reports as low as $100-120 \mathrm{mAs} .{ }^{14}$ Through a quality improvement program at our institution, we desired to achieve a 33\% reduction 
in CTP dose by reducing tube current to approximately $125 \mathrm{mAs}$ from our reference protocol performed at $190 \mathrm{mAs}$. The consequent changes in image quality, particularly from increased image noise, warranted further investigation before this protocol change was implemented. Furthermore, in recent years there has been the introduction of several postprocessing algorithms designed to reduce noise in CT scans acquired at lower doses. The threshold dose at which image quality changes significantly, and at which these algorithms could then subsequently add value, needs assessment. The purpose of this study was to use published techniques for noise addition to CT datasets to simulate dose-reduction to determine the subsequent effects on image quality and quantitative evaluation of CTP examinations.

\section{MATERIALS AND METHODS \\ Study Population}

In this case-control study, consecutive CTP examinations were retrospectively identified from an institutional review boardapproved, prospective clinical trial at our institution from August 2007-June 2010. Inclusion criteria for the prospective clinical trial were adult patients ( $\geq 18$ years) with documented aneurysmal subarachnoid hemorrhage at admission. Aneurysmal subarachnoid hemorrhage was diagnosed on initial noncontrast head CT, CSF analysis, CT angiography, and/or digital subtraction angiography. Exclusion criteria were a history of iodinated contrast allergy, renal impairment, or pregnancy.

\section{CTP Scanning Technique}

We performed CTP examinations by using a standard protocol at our institution, on LightSpeed or Pro-16 scanners (GE Healthcare, Milwaukee, Wisconsin), with cine 4i scanning mode and 45-s acquisition at 1 rotation per second by using 80 $\mathrm{kVp}$ and $190 \mathrm{mAs}$. CTDI-vol was $725.21 \mathrm{mGy}$, and the doselength product was $1450.42 \mathrm{mGy}-\mathrm{cm}$. Four sections, each at 5 -mm thickness, were acquired with the inferior extent at the basal ganglia, above the orbits, to minimize radiation exposure to the lenses. A total of $45 \mathrm{~mL}$ of low osmolar $(300 \mathrm{mg} / \mathrm{mL})$ or iso-osmolar $(320 \mathrm{mg} / \mathrm{mL})$ nonionic iodinated contrast was administered intravenously at $5 \mathrm{~mL} / \mathrm{s}$ by use of a power injector with a 5-s delay. CTP examinations from all participants were deidentified and were stored in the departmental research PACS in DICOM format. These nonmodified, nonsimulated, and acquired datasets are described in this study as the "reference" datasets.

\section{Assessment of the Relationship of Image Noise to Dose on Our Scanning Systems}

A well-understood relationship exists with image noise and dose, expressed as

$$
\text { noise } \propto 1 / \sqrt{\text { exposure }}
$$

in which noise at a given exposure level (E1) is defined as the $\mathrm{SD}, \sigma(E 1)$, of the CT numbers (HU) from a uniform ROI. The noise at a second exposure level (E2) can be predicted from a known exposure level E1 by the following relationship:

$$
\frac{\sigma(E 2)}{\sigma(E 1)}=\frac{\sqrt{E 1}}{\sqrt{E 2}}
$$

To test the validity of this relationship on our scanners, we scanned a $16-\mathrm{cm}$ polymethylmethacrylate cylinder phantom on a LightSpeed volumetric CT scanner at multiple tube currents ranging from the lowest to highest available settings (10 $\mathrm{mAs}-440 \mathrm{mAs}$ ) in 36 steps, otherwise by using the institutional-standard CTP parameters noted above. A single image was obtained at each tube current. Noise, defined as the SD of CT numbers within an ROI at the center of each image, was plotted against $1 / \sqrt{m A s}$. The near-linear relationship, shown in Online Fig 1, supports the concept that the addition of noise to CT images can predictably simulate dose reduction on our scanners. Please note that this noise model has already been established and validated, ${ }^{15}$ and thereby, our study is focused on evaluating the noise effects in CTP examinations performed at variable radiation doses.

\section{Noise Addition to Simulate Dose Reduction}

Britten et $\mathrm{al}^{15}$ demonstrated a technique, which we used in this study, to add spatially correlated statistical noise to the reconstructed (DICOM) reference CT images (before processing to generate perfusion maps), thereby simulating a lower-dose scan. These simulated datasets are described in this study as the "noise" datasets. The SD of the noise distribution to be added, $\sigma(a d d)$, to a scan at the reference exposure, E1, can be found by using the following relationship:

$$
\sigma^{2}(E 2)=\sigma^{2}(E 1)+\sigma^{2}(\text { add })
$$

An image-processing tool was developed on Matlab (MathWorks, Natick, Massachusetts) software capable of adding spatially correlated statistical noise to DICOM data in the manner shown by Britten et al. ${ }^{15}$ First, the spectral properties of CT noise were measured from the phantom data, and the noise power spectrum was calculated for each current setting. It was found that the shape of normalized noise power spectra did not vary significantly as a function of mAs, and we subsequently used the same spectral model for all cases and noise levels. The noise autocorrelation function was computed from these data, and its $11 \times 11$ window around the autocorrelation peak was saved as a convolution filter for generating colored noise in subsequent experiments at all noise levels. The $11 \times$ 11 filter was chosen instead of the $5 \times 5$ filter used by Britten et $\mathrm{al}^{15}$ because the autocorrelation function of noise was wider than the one they reported, with values reaching close to zero at a location 5 pixels away from the center as opposed to 2 pixels away.

To simulate noise with a proper power spectrum on an image, we then applied this filter to subsequent white Gaussian noise generated by Matlab and scaled to the desired SD. The noise was masked by a filter of valid pixels in the DICOM image and then was added to the same image. This procedure ensures that the noise spectrum of any simulated noise we add to any image will have the spectral properties observed in an actual CT scan of the phantom on this scanner.

$\sigma(E 1)$ at the reference exposure of $E 1=190 \mathrm{mAs}$ was determined as the average value of noise in an ROI placed in the frontal horn of the right or left lateral ventricle in the first cine image of the CTP reference dataset. Knowing E1, E2 (the desired simulated exposure), and $\sigma(E 1)$, calculation of $\sigma(E 2)$ is 

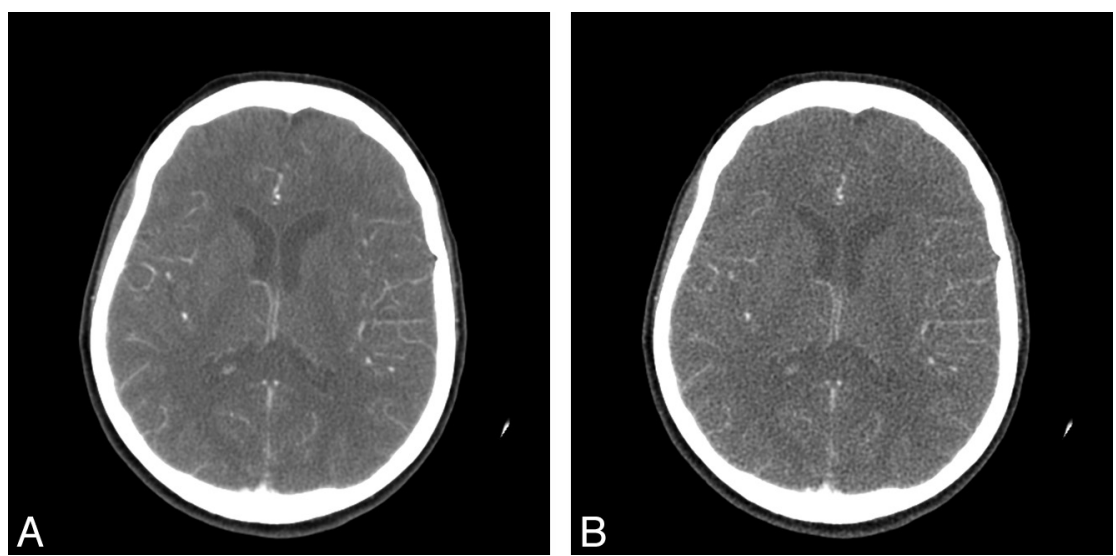

\section{CTP Postprocessing and Quantitative Analysis}

Postprocessing of the reference dataset and the 5 noise datasets into CBF, MTT, and $\mathrm{CBV}$ maps was performed on an Advantage Workstation by use of CTP software version 3.0 (GE HealthCare). This software uses a deconvolution method, which is considered most accurate for low-contrast injection rates. ${ }^{17}$ The postprocessing technique was standardized for all participants according to recommended guidelines, ${ }^{18}$ with the arterial input function as the

FIG 1. Representative reference scan at $190 \mathrm{mAs}(A)$ and simulated 44-mAs scan $(B)$ in a single section of a CTP examination. Dose reduction, achieved through tube current reduction, primarily results in increased image noise, demonstrated as increased "graininess" in the simulated 44-mAs scan.

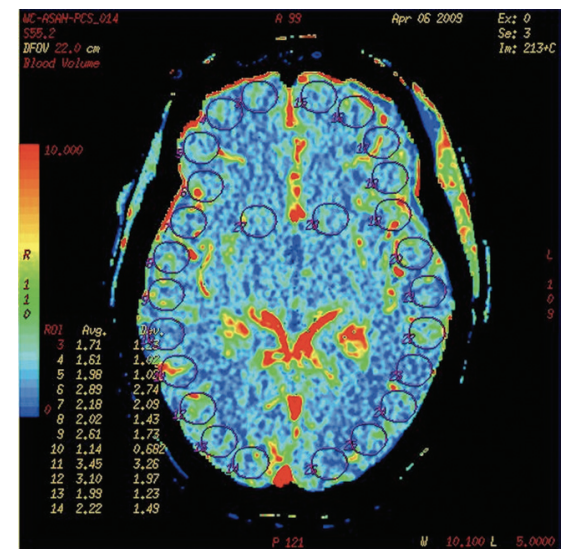

FIG 2. A representative blood volume map obtained in a single participant. The map shows placement of 26 uniform regions of interest: 12 in the right lobe, 12 in the left lobe, and 1 each over the right and left basal ganglia. Similar ROIs were placed over each section of a CTP dataset. The positions of ROIs from all sections in a given reference dataset were saved as a template and then were pasted onto the 5 generated noise datasets, ensuring perfect uniformity of position.

possible by using Equation 2. Knowing $\sigma(E 1)$ and $\sigma(E 2)$, calculation of $\sigma(a d d)$ is possible by using Equation 3 .

In this study, we use mAs as an approximation of exposure. Simulated exposure levels, E2, were chosen to represent a stepwise dose reduction down to approximately $125 \mathrm{mAs}$ to match previously published protocols highlighting low exposures in CTP examinations. ${ }^{14}$ An additional lower mAs scan, estimated at $44 \mathrm{mAs}$, was desired to match other studies by use of gel phantoms, rather than live human data, for CBF estimation. ${ }^{16}$

For each reference CTP DICOM dataset used as an input, the tool generated 5 datasets, each with a fixed amount of added noise distribution, $\sigma(a d d)$, at the following values: 0.8 , 2.0, 2.8, 5.3, and 13.7. We then wrote the noise-added images to a new DICOM file, keeping the same information in the original DICOM header, except for the series number, series instance Unique ID, and series description, and pushed back to the departmental research PACS, now as a separate series of the same study (Fig 1). Perfusion maps were then generated from these data. venous function were used for postprocessing each dataset in a given participant. No additional smoothing was performed.

For each participant, and within each section of the reference dataset, an investigator placed 26 ROIs that were standard 156$\mathrm{mm}^{2}$ size and round shape (12 in the right cerebral cortex, 12 in the left cerebral cortex, and 2 in the basal ganglia). These 26 ROIs per section were saved as a template and then were reapplied in the same exact positions on the corresponding sections within the 5 generated-noise datasets for the same participant (Fig 2). Therefore, there was exact matching of the ROI positions between the reference dataset and noise datasets for each participant. Between participants, ROI positions required minor adjustments only to ensure proper placement. Therefore, there was correspondence of ROI positions between participant scans as well (eg, ROI 1 was placed in the right frontal lobe of all scans, and ROI 26 was placed in the left basal ganglia of all scans). This enabled analysis of trends in perfusion parameters for each ROI at various noise levels across all scans (On-line Fig 2).

For every ROI, perfusion parameters (CBF, CBV, and MTT) were recorded as both colored image files (JPEG format) and DICOM files, which contained the raw pixel data. Values were recorded in text files. To measure variability and reproducibility in the perfusion parameters, noise datasets were generated 6 times at each noise level in 2 participants. These repeated-noise datasets are described as separate "trials" in this study.

In a subanalysis, a trained neuroradiologist (P.S.) with 12 years of experience reviewed perfusion maps of all reference datasets and identified ROIs with visual perfusion abnormalities, defined as focal areas with elevated MTT and/or decreased CBF.

\section{Qualitative Analysis of CTP}

Six radiologists with various levels of skill and training participated in the study to evaluate the image quality of the CTP maps. These included 2 board-certified neuroradiologists (one with 12 years of experience and the other with 17 years of experience), a diagnostic neuroradiology fellow with 6 years of experience, an interventional neuroradiology fellow with 6 years of experience, a board-certified body imaging radiologist with 9 years of experience, and a radiology resident with 2 years of experience. A 
software program was specifically developed for this study to allow the observer to compare the CTP maps generated from the 5 noise datasets to the CTP maps of the reference dataset in a random, blinded fashion. Each observer independently entered a quality score for each CTP map generated from the noise datasets. The quality score consisted of a 5-point Likert grading scale ( $1=$ equivalent image quality compared with the reference CTP map; 2 = slight inferior quality, no significant difference; $3=$ mild inferior quality, noticeable difference; $4=$ moderate inferior quality, significant difference; $5=$ severe inferior quality, poor image).

\section{Statistical Analysis}

The hypothesis for the quantitative evaluation of the CTP data was that each noise dataset (simulating reduced dose) had no change in perfusion parameters compared with the reference dataset, first across all ROIs, and second in a subanalysis of ROIs with visual perfusion abnormalities. On-line Figures $2 A-C$ showed that all ROIs had the same trends across noise levels. Therefore, it was considered statistically appropriate to analyze these quantitative data by using average values across all ROIs and sections for each participant. Because 6 datasets were available for each participant, repeated analysis (PROC MIXED) was performed with noise effect as a fixed effect and the correlation structure between subsequent measurements assumed as compound symmetry. ${ }^{20}$ The Dunnett method was adjusted for post hoc multiple comparisons. The differences of least square means in CTP parameters between noise datasets and reference datasets, across all ROIs and separately in ROIs with visual perfusion abnormalities, standard errors, unadjusted $P$ values, and adjusted $P$ values was reported. A coefficient of variance analysis, defined as the SD divided by the mean of the values multiplied by 100 , was used to determine the precision of the perfusion parameters in the 2 reference datasets from which the same noise datasets were generated 6 times (multiple trials).

In the qualitative evaluation, the relationship between the quality grade and noise level for each of the 3 perfusion maps, $\mathrm{CBF}, \mathrm{CBV}$, and MTT, was individually evaluated by use of the Spearman rank correlation coefficient. We calculated pairwise interuser agreeability for each map by using PABAK. ${ }^{21}$ Average of PABAK was then calculated to represent the interuser agreeability among 6 observers. We performed all statistical analyses by using SAS 9.2 (SAS Institute, Cary, North Carolina). All $P$ values were 2 -sided, and $P<.05$ was considered statistically significant.

\section{RESULTS}

A total of 22 CTP examinations from 22 participants (18 women, 4 men) were available at the start of this study from the prospective clinical trial, and all were included. The mean age was 55.0 years $\pm 13.5(\mathrm{SD})$, and the age range was $34-88$ years.

For 20 of the 22 participants, there existed [ 1 reference dataset/participant $) \times(1$ trial/reference dataset $) \times(4$ sections $/$ trial $) \times(26$ ROIs/section $)]+[(5$ noise datasets/participant $) \times(1$ trial/noise dataset $) \times(4$ sections $/$ trial $) \times(26 \mathrm{ROIs} /$ section $)]=$ 624 ROIs/participant. In 2 of the 22 participants, there existed [ 1 reference dataset/participant $) \times(1 \mathrm{trial} /$ reference dataset $) \times(4$ sections/trial $) \times(26$ ROIs/section $)]+([5$ noise datasets/partici-
Table 1: Simulated exposure for CTP examinations at each level of noise added

\begin{tabular}{ccc}
\hline Noise Level & $\begin{array}{c}\text { Added Noise Distribution, } \\
\boldsymbol{\sigma}(\text { add) }\end{array}$ & $\begin{array}{c}\text { Simulated Exposure, E2 } \\
\text { (Range) (mAs) }\end{array}$ \\
\hline 1 & 0.8 & $188(187-188)$ \\
2 & 2.0 & $177(174-180)$ \\
3 & 2.8 & $167(161-171)$ \\
4 & 5.3 & $127(116-135)$ \\
5 & 13.7 & $44(36-51)$ \\
\hline
\end{tabular}

Note:-The range is determined using noise values of the reference scans at $1 \mathrm{SD}$ above and below the mean, $\sigma(E 7)=7.5 \pm 0.8$.

E2 indicates second exposure level.

pant $) \times(6$ trials/noise dataset $) \times(4$ sections $/$ trial $) \times(26$ ROIs $/$ section) $]=3224$ ROIs/participant.

\section{Quantitative Assessment}

Noise measurements, as previously defined, obtained from an ROI in the frontal horn of the right or left lateral ventricles on the reference $\mathrm{CT}$ scans of the 22 participants yielded an average value of $7.48 \mathrm{HU}$ (SD, 0.84). Therefore, we report $\sigma(E 1)=7.5 \pm 0.8$. The simulated exposure, E2, at each level of added noise distribution, $\sigma(a d d)$ is calculated and is shown in Table 1 .

Differences in perfusion parameters across all ROIs and only in ROIs with visual perfusion abnormalities between the reference datasets and the noise datasets are shown in Table 2. These differences were small at all noise levels, with statistically significant differences present only at a simulated exposure of $44 \mathrm{mAs}$ when all ROIs were assessed.

The coefficient of variance of the perfusion parameters in the scans in which the same noise dataset was generated in 6 trials is shown in Table 3. As expected, the coefficient of variance values increase as the noise level increases, ranging from approximately $1 \%-7 \%$.

\section{Qualitative Assessment}

Twenty CTP datasets were included in the qualitative assessment, with a total of 1200 parametric CTP maps (400 CBF, $400 \mathrm{CBV}$, and $400 \mathrm{MTT}$ ) processed at 5 different noise levels. The colored map files in 2 of the datasets were corrupted and were therefore excluded from analysis. A total of 7200 quality scores were collected for analysis from each of the 6 observers. The frequency of the quality scores assigned by the 6 radiologists for the CBF, CBV, and MTT maps at each noise level is shown in Fig 3, with the Spearman rank coefficients and $P$ values. Statistically significant differences in the quality scores were assigned to the highest noise level 5 for the CBF, CBV, and MTT maps. We calculated the interobserver agreement by using the PABAK values for each CTP map (Table 4). Noise level 5 demonstrates the lowest agreement rates among the 6 observers, with mean PABAK scores for CBF, $\mathrm{CBV}$, and MTT of $0.52,0.83$, and 0.05 , respectively, representing poor to moderate agreement rates.

\section{DISCUSSION}

The goal of this study was to help guide a decision to reduce dose in CTP examinations at our institution from a reference protocol that used $80 \mathrm{keV}$ and $190 \mathrm{mAs}$. The target exposure of approximately $125 \mathrm{mAs}$ was selected based on other published work using this value, and represents a $33 \%$ reduction from the reference value. Before implementing this protocol change, we desired 
Table 2: Difference of least square means in perfusion parameters between reference dataset and noise datasets across all ROIs in all participants and across ROIs in participants with perfusion abnormalities (subanalysis)

\begin{tabular}{|c|c|c|c|c|c|c|c|c|c|}
\hline \multirow[b]{2}{*}{$\begin{array}{l}\text { Perfusion } \\
\text { Parameter }\end{array}$} & \multirow[b]{2}{*}{$\begin{array}{c}\text { Simulated } \\
\text { Exposure } \\
\text { (mAs) }\end{array}$} & \multicolumn{4}{|c|}{ All ROls } & \multicolumn{4}{|c|}{ Only in ROIs with Perfusion Abnormalities } \\
\hline & & $\begin{array}{c}\text { Difference } \\
\text { from } \\
\text { Reference } \\
\text { Dataset }\end{array}$ & $\begin{array}{l}\text { Standard } \\
\text { Error }\end{array}$ & $P$ & Adj $P$ & $\begin{array}{c}\text { Difference } \\
\text { from } \\
\text { Reference } \\
\text { Dataset }\end{array}$ & $\begin{array}{l}\text { Standard } \\
\text { Error }\end{array}$ & $P$ & Adj $P$ \\
\hline \multirow[t]{5}{*}{$\mathrm{CBF}(\mathrm{mL} / 100 \mathrm{~g} / \mathrm{min})$} & 188 & -0.12 & 0.68 & .87 & $>.99$ & -0.35 & 0.86 & 69 & $>.99$ \\
\hline & 177 & -0.20 & 0.68 & .77 & $>.99$ & -0.22 & 0.86 & .80 & $>.99$ \\
\hline & 167 & -0.72 & 0.68 & .29 & .73 & -0.67 & 0.86 & .44 & .90 \\
\hline & 127 & -0.60 & 0.68 & .38 & .85 & -0.01 & 0.86 & .99 & $>.99$ \\
\hline & 44 & -1.81 & 0.68 & .01 & .04 & 0.47 & 0.86 & .58 & .97 \\
\hline \multirow[t]{5}{*}{ CBV $(\mathrm{mL} / 100 \mathrm{~g} / \mathrm{min})$} & 188 & 0.00 & 0.02 & .89 & $>.99$ & -0.01 & 0.02 & .74 & $>.99$ \\
\hline & 177 & 0.00 & 0.02 & .91 & $>.99$ & 0.00 & 0.02 & .99 & $>.99$ \\
\hline & 167 & 0.00 & 0.02 & .92 & $>.99$ & 0.00 & 0.02 & .93 & $>.99$ \\
\hline & 127 & 0.02 & 0.02 & .26 & .68 & 0.01 & 0.02 & .62 & .98 \\
\hline & 44 & 0.07 & 0.02 & $<.0001$ & $<.001$ & 0.10 & 0.02 & $<.0001$ & $<.0001$ \\
\hline \multirow[t]{5}{*}{ MTT (sec) } & 188 & 0.00 & 0.06 & .94 & $>.99$ & 0.03 & 0.10 & .79 & $>.99$ \\
\hline & 177 & 0.02 & 0.06 & .74 & $>.99$ & 0.05 & 0.10 & .66 & $>.99$ \\
\hline & 167 & 0.06 & 0.06 & .32 & .78 & 0.07 & 0.10 & .47 & .92 \\
\hline & 127 & 0.11 & 0.06 & .08 & .28 & 0.07 & 0.10 & .51 & .95 \\
\hline & 44 & 0.46 & 0.06 & $<.0001$ & $<.001$ & 0.33 & 0.10 & .00 & .01 \\
\hline
\end{tabular}

Note:-ROls with perfusion abnormalities were identified by a trained neuroradiologist (P.S.) with 12 years of experience. Adj $P$ indicates adjusted $P$ value.

Table 3: Coefficient of variance of perfusion parameters in noise datasets generated in 6 trials at each noise level

\begin{tabular}{cccc}
\hline & \multicolumn{3}{c}{ Coefficient of Variance (\%) } \\
\cline { 2 - 4 } Simulated Exposure (mAs) & CBF & CBV & MTT \\
\hline 188 & 1.3 & 0.3 & 1.2 \\
177 & 2.1 & 0.7 & 1.9 \\
167 & 3.2 & 0.9 & 2.9 \\
127 & 4.1 & 1.9 & 4.6 \\
44 & 7.1 & 4.5 & 6.7 \\
\hline
\end{tabular}

Note:-Coefficient of variance is defined as SD/mean $\times 100$

some evidence to present to both our interpreting radiologists as well as to referring clinicians that this level of dose reduction could be attempted without differences in the quality of the images or quantitative data. Using previously published techniques, we generated simulations of reduced-dose CT scans at several incremental values down to $125 \mathrm{mAs}$. An additional simulation at a markedly reduced dose of $44 \mathrm{mAs}$, based on prior studies by use of phantom data, ${ }^{16}$ was included as an attempt to determine a lower limit to design future studies.

Radiologists, working independently of one another, documented no statistically significant differences in the quality of the perfusion maps between those generated from the reference scans and those generated from the reduced-dose simulations, down to values of $127 \mathrm{mAs}$. On maps generated from simulated $44 \mathrm{mAs}$ scans, however, there were statistically significant reductions in quality and agreement rates, raising the concern of increased variability in interpretation of CTP examinations as the dose is reduced to this level.

The dose reduction simulation and quantitative analysis were performed independent of the qualitative analysis in this study, by a different group of investigators. The results of these analyses, however, were complementary. CBF, CBV, and MTT values showed no statistically significant differences from the reference values at simulated exposures down to $127 \mathrm{mAs}$, but differences did become significant at the lowest simulated exposure for all perfusion maps. Furthermore, the coefficient of variance of these values was less than $5 \%$ at all simulated exposure levels except the lowest, suggesting that the reproducibility of these values may be compromised as the dose is markedly reduced. The true inflection of statistical significance in our study is likely to occur between $127 \mathrm{mAs}$ and $44 \mathrm{mAs}$. The clinical significance of determining the exact statistical inflection point may be a subject of debate, as discussed below.

Although statistically significant differences of CTP parameters from reference values were shown at the lowest simulated exposure level, these differences were very small and may not be clinically significant. This result held true when assessing all ROIs and only ROIs with visual perfusion abnormalities. This clinical significance is best understood in the context of the threshold values in perfusion parameters that distinguish normal from abnormal, still a subject of debate in the CTP community. Nevertheless, the small changes in these parameters are an important result. Some recently introduced strategies to reduce dose in CTP and in other CT examinations include postprocessing algorithms aimed at reducing noise in low-dose examinations. ${ }^{16,22}$ Krissak et $\mathrm{al}^{22}$ used a highly constrained back-projection local reconstruction postprocessing technique on CTP images obtained at $30 \mathrm{mAs}$ compared with their standard $200 \mathrm{mAs}$ in patients with acute stroke and demonstrated no difference in image quality or mean attenuation within an ROI between these examinations. Using a different postprocessing technique of nonlinear diffusion filtering, Saito et $\mathrm{al}^{16}$ demonstrated by using gel phantoms that CBF values acquired at $50 \mathrm{mAs}$ were similar to the reference values acquired at $200 \mathrm{mAs}$. It would be highly informative if these prior studies had reported the results of CTP analysis on data before undergoing these postprocessing techniques. These postprocessing techniques will be most valuable if CTP examinations acquired at these low doses result in statistically and clinically significant differences in perfusion parameters. If, as our simulated data suggest, these differences in both qualitative and quantitative interpretation are small and perhaps clinically insignificant, then 

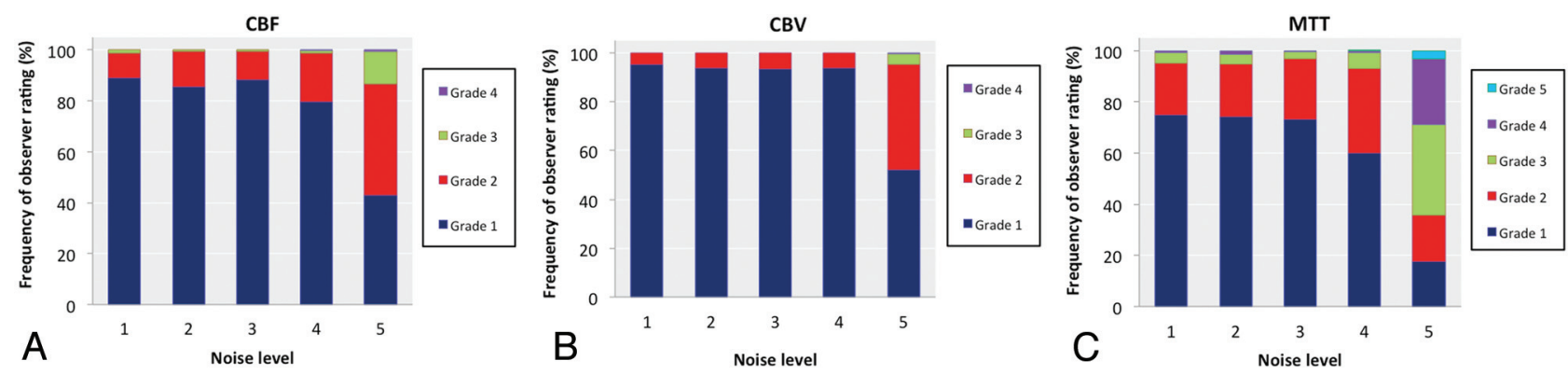

FIG 3. Frequency of quality scores assigned by the 6 observers to noise datasets at each noise level for $(A) C B F(S p e a r m a n$ rank coefficient $=-0.34 ; P<.0001$ ), (B) CBV (Spearman rank coefficient $=-0.35 ; P<.0001$ ), and $(C)$ MTT (Spearman rank coefficient $=-0.44$; $P<.0001)$

Table 4: Mean calculated interuser agreeability PABAK score for each CTP map

\begin{tabular}{cccc}
$\begin{array}{c}\text { Simulated Exposure } \\
\text { (mAs) }\end{array}$ & $\begin{array}{c}\text { Mean PABAK Score of } \\
\text { CBF Maps (range) }\end{array}$ & $\begin{array}{c}\text { Mean PABAK Score of } \\
\text { CBV Maps (range) }\end{array}$ & $\begin{array}{c}\text { Mean PABAK Score of } \\
\text { MTT Maps (range) }\end{array}$ \\
\hline 188 & $0.95(0.88-1)$ & $1.00(1-1)$ & $0.83(0.70-0.98)$ \\
177 & $0.98(0.93-1)$ & $1.00(1-1)$ & $0.86(0.70-0.98)$ \\
167 & $0.98(0.93-1)$ & $1.00(1-1)$ & $0.88(0.73-1)$ \\
127 & $0.95(0.88-1)$ & $1.00(1-1)$ & $0.74(0.43-1)$ \\
44 & $0.51(0.18-0.93)$ & $0.83(0.58-1)$ & $0.05(-0.87-0.68)$ \\
\hline
\end{tabular}

We did not see this kind of behavior in our phantom scans, and we did not know of a physical mechanism by which additive noise levels should affect the noise spectrum. The exact numeric relationship between dosage and noise may vary somewhat among scanners, but we believe that the behavior of CTP maps we have cataloged should continue to hold across the value of postprocessing techniques and their dose range of necessity needs re-evaluation. Our study supports the possibility that dose reduction can sometimes be achieved with reduction in $\mathrm{mAs}$ alone, without the need for additional postprocessing algorithms. Large-scale, prospective trials that obtain true, nonsimulated data would be of tremendous value not only to assess the validity of our simulations, but also to assess the extent to which dose can truly be reduced in a variety of CT examinations.

The simulations and analysis performed in this study suggest that a 33\% dose reduction may be possible on CTP examinations performed at our institution at reference settings of $80 \mathrm{keV}$ and 190 mAs, without observed qualitative or quantitative differences in perfusion maps. These data should be interpreted with some caution and help only to inform future studies assessing these differences by using true, nonsimulated data. The value of studies that use simulations is that they can be performed without the radiation risk incurred when performing true, repeated CT scans on patients.

One of the limitations of our study was the method used for adding noise to simulate different exposure levels. Noise addition for this purpose is ideally performed on the projection data, as reported by Mayo et al. ${ }^{23}$ However, these raw projection data are rarely stored for long periods, making retrospective studies such as this difficult to perform. Britten et $\mathrm{al}^{15}$ attempted to show that noise addition to reconstructed data could achieve similar results as noise addition to raw data, but it should be recognized that validation of the technique was based on a subjective comparison of acquired low-dose and simulated low-dose images. Our study is based on the assumption that this validation holds true even for quantitative assessments, but this assumption may need further validation.

Limitations also included the possibility of variations in the noise spectrum with reduced dose in the same scanner and among scanners. A priori, we saw little reason to believe that the noise spectrum should change because of dosage on the same scanner. scanners. We did use a calibration strategy to learn the correct numeric factors for a given scanner, and that strategy can be applied to other scanners when attempting to repeat the study. Such repeated studies, we believe, will show a very limited dose dependency on quantitative CTP parameters.

It may also be noted that many centers are already using lower tube currents in their CTP protocols on 64- and 128-detector scanners. Although reference image noise levels may vary among protocols on these different machines, the relationship between noise and exposure (Equation 1) should continue to hold true. Our study identifies a very limited dose dependency in CTP parameters within a certain dose range. At any given site that has recently changed its CTP protocol to achieve lower dose through modest tube current reduction, our study suggests that CTP parameters between the 2 protocols may be comparable, even without the use of postprocessing algorithms. Although further validation is still needed, this result may have important implications in clinical research using these quantitative data.

\section{CONCLUSIONS}

On the basis of dose-reduction simulation techniques, this study supports the potential of a 33\% reduction in dose on CTP examinations performed at $80 \mathrm{keV}$ and $190 \mathrm{mAs}$ without statistically significant differences in quantitative perfusion parameters or image quality. Reduced image quality and increased variability were observed at the lowest simulated dose ( $44 \mathrm{mAs}$ ) for all perfusion maps, raising concern for variability in interpretations at this level. Nevertheless, quantitative differences were small, highlighting a very limited dose dependency in CTP parameters within a certain dose range. Future studies should investigate the clinical significance of these changes and should reassess the need for postprocessing algorithms aimed at reducing noise in low-dose CT examinations. Because this study used simulations of dose reduction, results should be interpreted with caution but nonetheless can be used to inform future prospective studies that ac- 
quire true, nonsimulated, CTP scans performed at a reduced radiation dose.

\section{ACKNOWLEDGMENTS}

The authors thank Ya-lin Chiu, MS, for her contributions to the biostatistical analysis in this paper.

Disclosures: Krishna Juluru, Ashish Raj, Holly Delaney, Edward Greenberg, Charles Herrmann, Brian Liu, Noor Ahmed Al-Khori-RELATED: Grant: NIH, ${ }^{*}$ Comments: This research was made possible by Grant No. 5K23NS058387-02 from the National Institute of Neurological Disorders and Stroke (NINDS), a component of the National Institutes of Health (NIH). The contents of this study do not necessarily represent the official views of the NINDS or the NIH. Pina Sanelli-RELATED: Grant: NIH K23 award; ${ }^{*}$ UNRELATED: Grants/Grants Pending: NIH K23 award. ( ${ }^{*}$ Money paid to institution.)

\section{REFERENCES}

1. Provenzale JM, Shah K, Patel U, et al. Systematic review of CT and MR perfusion imaging for assessment of acute cerebrovascular disease. AJNR Am J Neuroradiol 2008;29:1476-82

2. Turk A, Magarik JA, Chaudry I, et al. CT perfusion-guided patient selection for endovascular treatment of acute ischemic stroke is safe and effective. J Neurointerv Surg 2011;4:261-65

3. Konstas AA, Wintermark M, Lev MH. CT perfusion imaging in acute stroke. Neuroimaging Clin N Am 2011;21:215-38, ix.

4. Suzuki K, Morita S, Masukawa A, et al. Utility of CT perfusion with 64-row multi-detector CT for acute ischemic brain stroke. Emerg Radiol 2011;18:95-101

5. Loftus ML, Minkowitz S, Tsiouris AJ, et al. Utilization guidelines for reducing radiation exposure in the evaluation of aneurysmal subarachnoid hemorrhage: a practice quality improvement project. AJR Am J Roentgenol 2010;195:176-80

6. Sanelli PC, Jou A, Gold R, et al. Using CT perfusion during the early baseline period in aneurysmal subarachnoid hemorrhage to assess for development of vasospasm. Neuroradiology 2011;53:425-34

7. Wintermark M, Ko NU, Smith WS, et al. Vasospasm after subarachnoid hemorrhage: utility of perfusion CT and CT angiography on diagnosis and management. AJNR Am J Neuroradiol 2006;27:26-34

8. Sanelli PC, Ugorec I, Johnson CE, et al. Using quantitative CT perfusion for evaluation of delayed cerebral ischemia following aneurysmal subarachnoid hemorrhage. AJNR Am J Neuroradiol 2011;32:2047-53

9. Ramli N, Rahmat K, Mah E, et al. Use of permeability surface areaproduct to differentiate intracranial tumours from abscess. Biomed Imaging Interv J 2009;5:e6

10. Sergides I, Hussain Z, Naik S, et al. Utilization of dynamic CT perfu- sion in the study of intracranial meningiomas and their surrounding tissue. Neurol Res 2009;31:84-89

11. Ding B, Ling HW, Chen KM, et al. Comparison of cerebral blood volume and permeability in preoperative grading of intracranial glioma using CT perfusion imaging. Neuroradiology 2006;48:773-81

12. Fainardi E, Di Biase F, Borrelli M, et al. Potential role of CT perfusion parameters in the identification of solitary intra-axial brain tumor grading. Acta Neurochir Suppl 2010;106:283-87

13. Smith AB, Dillon WP, Gould R, et al. Radiation dose-reduction strategies for neuroradiology CT protocols. AJNR Am J Neuroradiol 2007;28:1628-32

14. Wintermark M, Smith WS, Ko NU, et al. Dynamic perfusion CT: optimizing the temporal resolution and contrast volume for calculation of perfusion CT parameters in stroke patients. AJNR Am J Neuroradiol 2004;25:720-29

15. Britten AJ, Crotty M, Kiremidjian H, et al. The addition of computer simulated noise to investigate radiation dose and image quality in images with spatial correlation of statistical noise: an example application to x-ray CT of the brain. Br J Radiol 2004;77:323-28

16. Saito N, Kudo K, Sasaki T, et al. Realization of reliable cerebralblood-flow maps from low-dose CT perfusion images by statistical noise reduction using nonlinear diffusion filtering. Radiol Phys Technol 2008;1:62-74

17. Wintermark M, Maeder P, Thiran JP, et al. Quantitative assessment of regional cerebral blood flows by perfusion CT studies at low injection rates: a critical review of the underlying theoretical models. Eur Radiol 2001;11:1220-30

18. Sanelli PC, Lev MH, Eastwood JD, et al. The effect of varying userselected input parameters on quantitative values in CT perfusion maps. Acad Radiol 2004;11:1085-92

19. Wintermark M, Lau BC, Chien J, et al. The anterior cerebral artery is an appropriate arterial input function for perfusion-CT processing in patients with acute stroke. Neuroradiology 2008;50:227-36

20. Liang K-Y, Zeger SL. Longitudinal data analysis using generalized linear models. Biometrika 1986;73:13-22

21. Cunningham M. More than Just the Kappa Coefficient: A Program to Fully Characterize Inter-Rater Reliability between Two Raters [Internet]. SAS Global Forum 2009. 2009 [cited 2012 May 22]; Available from: http://support.sas.com/resources/papers/proceedings09/ 242-2009.pdf

22. Krissak R, Mistretta CA, Henzler T, et al. Noise reduction and image quality improvement of low dose and ultra low dose brain perfusion CT by HYPR-LR processing. PloS One 2011;6:e17098

23. Mayo JR, Hartman TE, Lee KS, et al. CT of the chest: minimal tube current required for good image quality with the least radiation dose. AJR Am J Roentgenol 1995;164:603-07 